\title{
IMPUESTO A LA RENTA DE PROFESIONALES INDEPENDIENTES EN RELACiÓn CON los PRINCIPIOS DE EQUidad y Justicia en el Perú
}

\section{INCOME TAX PROFESSIONALS INDEPENDENT IN RELATION TO THE PRINCIPLES OF EQUITY AND JUSTICE IN PERU}

\author{
Nazareth Ruth Velásquez Peralta*
}

\begin{abstract}
RESUMEN
T a presente investigación tiene por objetivo general demostrar que el impuesto a la ren-

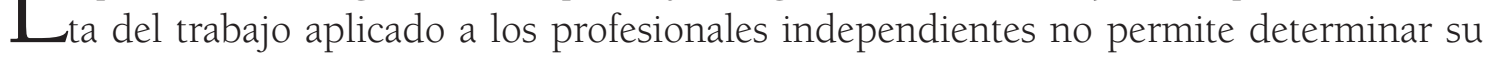
real capacidad contributiva dentro del principio de equidad y justicia en el Perú. El diseño de la investigación fue no experimental, correlacional y de nivel cuantitativo. La información se recabó de una muestra de 50 profesionales independientes afiliados al Colegio de Contadores Públicos de Áncash, escogida de manera dirigida de entre una población de 670 matriculados al 31 de diciembre del 2005. A ellos se les aplicó un cuestionario mediante la técnica de la encuesta y se obtuvieron los siguientes resultados: del total de la muestra tomada, el $56 \%$ de los profesionales tiene una renta neta imponible bajo un sistema de deducción real, el $38 \%$ de los profesionales tiene un ingreso promedio que alcanza el primer tramo imponible, un $4 \%$ alcanza el segundo tramo imponible y solo el $2 \%$ alcanza el tercer tramo imponible. Finalmente se ha comprobado que el impuesto a la renta de cuarta categoría que se aplica en el Perú no permite determinar la real capacidad contributiva de los profesionales, por lo que se incurre en injusticia e inequidad, de modo que la hipótesis planteada se demuestra como verdadera.
\end{abstract}

Palabras claves: impuesto a la renta, equidad y justicia, capacidad contributiva y profesionales independientes.

* Contador público colegiado, magíster en tributación, doctor en Contabilidad y Finanzas de la Universidad Nacional de Trujillo. Docente contratado de pregrado y posgrado por la Universidad Católica Los Ángeles de Chimbote. Auditor independiente y asesor de empresas en lo contable, tributario y laboral. 


\begin{abstract}
The present study was intended to demonstrate that the overall tax on labor income applied to independent professionals can not determine their real ability in the principle of fairness and justice in Peru. The research design was not experimental, correlational and quantitative level. The information was collected from a sample of 50 independent professionals affiliated with the Institute of Chartered Accountants of Ancash, chosen in a targeted manner from a population of 670 enrolled at December 31, 2005, we applied a questionnaire by the survey technique, and the following results were obtained: the total of the sample, $56 \%$ of professionals have a net taxable income under actual deduction system, $38 \%$ of professionals have an average income that reaches the first taxable stretch a $4 \%$ tax reaches the second stage and only $2 \%$ tax reaches the third stage. Finally, it was found that the Income Tax fourth category that applies in Peru can not determine the real ability of professionals so incurred injustice and inequality and can prove the hypothesis as true.
\end{abstract}

Keywords: income tax, equality and justice, independent capacity and independent professionals. 


\section{INTRODUCCIÓN}

El impuesto a la renta de los profesionales independientes que se encuentra comprendida en el universo de categorías de rentas de personas naturales debería ser equitativo y progresivo; sin embargo, nuestra legislación actual no contempla una adecuada determinación de la renta neta imponible en el marco de la capacidad contributiva de estos contribuyentes, toda vez que se ve distorsionada por insuficientes tramos de ingresos imponibles establecidos y porque están enmarcados en un sistema de deducción de gasto que no es real, de modo que deviene en un impuesto muy injusto en nuestra sociedad.

Las reformas tributarias que se han dado en el tiempo fundamentalmente tuvieron su origen en la necesidad de lograr la suficiencia recaudatoria que permita, sobre todo, afrontar las presiones de gasto, simplificar el sistema tributario y avanzar en mejorar su equidad. En ese sentido, la actual coyuntura de expansión de los recursos fiscales debe funcionar no como un desacelerador de la reforma tributaria, sino más bien como una base para impulsar un incremento sostenido de la recaudación, que permita a la vez concientizar a los trabajadores independientes para que contribuyan con el Estado de acuerdo a sus posibilidades.

La Constitución Política del Perú (1993), en el artículo 74, precisa que las normas tributarias se rigen por ciertos principios del derecho, como el de legalidad, igualdad, respeto a los derechos fundamentales de la persona y la no confiscatoriedad; por lo tanto, el legislador debe individualizar la carga tributaria del contribuyente, y valorar su situación personal y familiar, llamada aspecto subjetivo del principio de la 'capacidad contributiva'. Es importante que los impuestos que recaigan sobre los contribuyentes contemplen, unos más que otros, ambos aspectos del principio de capacidad contributiva, de lo contrario produciría una normativa adversa a los lineamientos de la justicia.

El Decreto Legislativo N. ${ }^{\circ} 972$ (2007), cuyo objetivo fue regular el tratamiento de las normas de rentas de capital, modifica el tratamiento que la ley del impuesto a la renta (LIR) otorga a la persona natural. En tal sentido, a partir del 1 de enero del 2009, se eliminó la tributación tomando como base imponible la renta neta anual, y existirá una nueva conformación de rentas de acuerdo a los siguientes niveles:

a. Renta de capital: que agrupa a la primera y segunda categoría

b. Rentas empresariales: que corresponde a la tercera categoría

c. Rentas de trabajo: que agrupa a la cuarta y quinta categoría

Millán (1999) señala que la capacidad contributiva surge después de que el sujeto pueda satisfacer sus necesidades primarias, como la alimentación, el vestido, la vivienda, la sanidad, la educación, por señalar a las más indispensables; entonces el contribuyente será contribuyente apto. 
Sin duda es sumamente importante respetar las necesidades primarias de tantas personas, sobre todo de aquellas que no reciben los beneficios propios de trabajar en forma dependiente para empresas formalmente constituidas; sin embargo realizan trabajo independiente para subsistir. Por ello el Estado debe considerar en sus políticas el perjuicio y atraso que supone para muchas familias este escenario laboral.

Por lo expuesto, el enunciado del problema de investigación es el siguiente: ¿el impuesto a la renta de profesionales independientes aplicado en el Perú permite determinar la real capacidad contributiva en relación con los principios de equidad y justicia? Para responder este enunciado se ha establecido el siguiente objetivo principal: demostrar que el impuesto a la renta de cuarta categoría aplicado en el Perú que lo integran los profesionales independientes no permite determinar la real capacidad contributiva de los profesionales dentro de los principios de equidad y justicia. Para obtener el objetivo general se plantearon los siguientes objetivos específicos: 1. determinar cuáles son las deducciones establecidas para las rentas de cuarta categoría, 2. analizar la incidencia del pago de aquel impuesto sobre la capacidad contributiva de los profesionales independientes, 3. analizar la incidencia de la norma tributaria en el grado de la informalidad de los profesionales independientes y 4 . proponer alternativas de reformas en las deducciones para las rentas de cuarta categoría.

La justificación del presente trabajo de investigación tiene la imperiosa necesidad de demostrar que el impuesto a la renta en los profesionales independientes es injusto e inconstitucional; asimismo, plantear alternativas de solución para encaminar a esta categoría de impuesto hacia la equidad tributaria tomando en cuenta el principio de la capacidad contributiva y los aspectos legales vigentes.

Además se proponen alternativas necesarias para una reforma del impuesto a las rentas de cuarta categoría bajo los principios de equidad, justicia y capacidad contributiva considerar como instrumento a la política familiar, eliminar la deducción flat, establecer el sistema de deducción al detalle (gastos reales de la familia) y aumentar la escala progresiva con mayores tramos imponibles.

\section{MATERIALES Y MÉTODOS}

La investigación fue de tipo cuantitativo y nivel descriptivo. Para realizarla se aplicó un cuestionario de preguntas cerradas a una muestra poblacional de cincuenta profesionales independientes afiliados al Colegio de Contadores Públicos de Áncash. El diseño de la investigación fue no experimental-descriptivo. Para recoger la información se aplicó la técnica de la encuesta, para lo cual se coordinó previamente con los profesionales independientes del ámbito de estudio el día y la hora de la entrevista. 


\section{RESULTADOS}

Mediante un balance crítico del marco doctrinario y constitucional del impuesto a la renta en los profesionales independientes contra la legislación actual y no consideración de los principios constitucionales, se puede llegar a determinar los resultados a través de las encuestas realizadas a ciertos profesionales independientes.

Respecto al objetivo específico 2, para una mejor explicación se segmentó a los profesionales encuestados en tres grupos (ver anexos Tabla 1 y Gráfico 1):

- Grupo 1: representa el 56 \% del total de la muestra; es el grupo de profesionales independientes cuyas rentas no alcanzaron el monto imponible mínimo de acuerdo a ley.

- Grupo 2: representa un 38 \% del total de la muestra; es el grupo de profesionales cuyas rentas alcanzaron el primer tramo imponible con una tasa impositiva del 15 $\%$, que se aplicará a la renta neta para determinar la deuda, y cuyos ingresos no son mayores a las 27 unidades impositivas tributarias (UIT).

- Grupo 3: representa un 4 \% del total de la muestra; es el grupo de profesionales cuyas rentas alcanzan el segundo tramo imponible para determinar su deuda. Se consideran las tasas impositivas del $15 \%$ y $21 \%$ de la renta neta, que pertenecen a la segunda escala de acuerdo a sus ingresos por exceso de 27 UIT.

- Grupo 4: representa un 2 \% del total de la muestra; es el grupo de profesionales cuyas rentas alcanzan el tercer tramo imponible para determinar su deuda. Se consideran las tasas del 15 \%, 21 \% y 30 \% según los tramos superados, respectivamente, que pertenecen a la tercera escala de acuerdo a sus ingresos por exceso de 54 UIT.

Respecto al objetivo específico 4 , se proponen y analizan alternativas de reforma del impuesto a las rentas de cuarta categoría (ver cuadros 1, 2, 3 y 4).

\section{DISCUSIÓN}

Respecto al objetivo específico 2:

- El grueso de las rentas de los profesionales en estudio no alcanza el mínimo imponible establecido por ley (56 \%). Estos resultados confirman la realidad laboral y social en nuestro país, puesto que se correlacionan con los alarmantes índices de desempleo, altas tasas de informalidad laboral, elevados indicadores de insatisfacción laboral (en formas de contratación, niveles remunerativos y acceso a beneficios) y exigua aprobación a las políticas laborales del gobierno en curso, que no consideran en sus planes estratégicos estatales la realidad problemática de los trabajadores independientes.

- $\quad$ Por lo menos, un 38 \% de las rentas de los profesionales en estudio alcanza el primer tramo imponible para rentas de cuarta categoría. Si se contrastan estos resultados con 
la información publicada por la Superintendencia Nacional de Aduanas y de Administración Tributaria (Sunat), se tiene que este resultado es mayor a la media nacional de rentas por personas naturales (22 \%), de las cuales poco más del 68 \% provenía de los ingresos de trabajadores independientes; sin embargo, de la investigación se observó que -en el caso de los profesionales en estudio- aquellas rentas se encontraban ligeramente por encima del límite inferior del primer tramo imponible.

- Apenas un 4 \% de las rentas de los profesionales en estudio alcanzó el segundo tramo de renta imponible, en tanto que solo el 2 \% de ellos alcanzó el tercer tramo imponible. Asimismo trascendió de la investigación a estos profesionales que, tal y como está diseñada la norma vigente, las deducciones permitidas no reflejan la real situación económica y financiera de las unidades familiares de cada profesional independiente, quienes justamente por razones de su elevada carga familiar (la que incorpora gastos académicos, gastos de salud, gastos recreativos, entre otros) se ven en la imperiosa necesidad de realizar más de una actividad laboral, la cual no necesariamente es tomada en las condiciones que el contribuyente espera (formal, en planilla y con beneficios sociales), sino que es reiterativa en la actividad independiente, la que a su vez suma sus ingresos de cuarta categoría, de modo que le corresponde pagar mayor impuesto a la renta por alcanzar los tramos imponibles más altos.

Respecto al objetivo específico 4:

El análisis propuesto y realizado en los cuadros del 1 al 4 revela que, en el caso de profesionales independientes con carga familiar y sin ella, con gastos comunes reales que no superen el monto mínimo mensual imponible y dadas las deducciones de ley, el fisco no recaudaría nada según como están estructurados los tramos (hasta 15 UIT, entre 15 y 27 UIT, más de 27 UIT) y tasas impositivas (15\%, 21 \% y $30 \%$ ); sin embargo, en ese mismo escenario de rentas y gastos - de profesionales independientes con carga familiar y sin ella-, pero en el que se practiquen modificaciones tributarias y se incorporen más tramos al mismo tiempo que se reducen los límites imponibles y junto con ellos las tasas impositivas, entonces el fisco lograría recaudar donde antes había.

Por otro lado, en contrapartida con lo analizado en el párrafo anterior, se tiene que en un escenario de profesionales independientes con carga familiar y sin ella, con gastos comunes reales que superen el monto mínimo mensual imponible y dadas las deducciones de ley, pero con sistema tributario que haya incorporado más tramos, reducidos los límites imponibles y junto con ellos las tasas impositivas, entonces continuaría recaudando de tales contribuyentes, aunque si bien es cierto no en los mismos niveles que en un sistema impositivo como el vigente, pero sí con una potencial mejora en la percepción de estar frente a un impuesto aplicado con equidad y justicia, lo que de cualquier manera incide en la disposición del contribuyente a cumplir con sus obligaciones tributarias y a minimizar riesgos de informalidad, evasión, etc. 


\section{CONCLUSIONES}

Respecto al objetivo específico 1, la investigación llegó a las siguientes conclusiones:

1. Se determinó la existencia de profesionales independientes que tienen capacidad contributiva, pero que con el sistema de deducción flat no contribuyen al fisco.

2. Se determinó la existencia de profesionales independientes cuyos gastos reales superan sus ingresos.

Respecto al objetivo específico 2, la investigación llegó a las siguientes conclusiones:

1. Se demuestra que la legislación vigente no aplica la verdadera capacidad contributiva, lo que genera un impuesto confiscatorio.

2. Se demuestra que la actual norma del impuesto a la renta de profesionales independientes no considera los principios establecidos en el artículo 74 de nuestra Constitución Política, por lo tanto es inconstitucional.

Respecto al objetivo específico 3, la investigación llegó a las siguientes conclusiones:

1. Existen problemas cuando se aplican los principios de capacidad contributiva, igualdad y progresividad a la persona natural.

2. Existen algunos problemas por resolver en materia tributaria que colisionan con la protección a la familia que la Constitución norma.

Respecto al objetivo específico 4, se proponen las siguientes alternativas de reforma del impuesto a las rentas de cuarta categoría:

1. Eliminar el sistema de deducción de gastos flat y establecer un sistema de deducción de gastos al detalle, de modo que así se recupera el principio de causalidad en esta categoría de rentas:

- $\quad$ 1. ${ }^{a}$ propuesta: la creación de un sistema de gastos al detalle deberá comprender el reconocimiento de deducciones personales y familiares del contribuyente.

2. El sistema de deducciones al detalle se debe reglamentar para evitar distorsiones intencionales:

- 2. ${ }^{a}$ propuesta: se deberían eliminar los sistemas de retenciones para rentas de personas naturales, tanto de cuarta categoría como de quinta estableciendo un sistema de pago a cuenta.

De no considerarse la propuesta planteada, tener en cuenta la siguiente:

- $\quad$ 3. ${ }^{a}$ propuesta: ampliar las escalas de los tramos imponibles vigentes. 
Finalmente se ha comprobado que, de acuerdo a los resultados obtenidos, la legislación vigente del impuesto a la renta de los profesionales independientes no cumple con ninguno de los principios de equidad, justicia ni capacidad contributiva establecidos en la Constitución Política del Perú, con lo que la hipótesis planteada se demuestra como verdadera.

\section{REFERENCIAS BIBLIOGRÁFICAS}

Centro Interamericano de Administraciones Tributarias (CIAT) (s/f). Disponible en: www. ciat.org.

Constitución Política del Perú (1993). Lima, Perú.

Cosciani, C. (S/F). La acumulación de las rentas de los cónyuges. La información (tomo XXXII), p. 1625.

Decreto Legislativo N. 972 (2007). Lima, Perú.

García, M. (2000). El principio de capacidad contributiva como criterio esencial para una reforma fiscal. Universidad de Salamanca.

García R. (1980). Teoría y técnica del impuesto a la renta. Santo Domingo.

Giannini, A. (1957). Instituciones de derecho tributario. (n. ${ }^{\circ}$ 18). Madrid.

González, F. (s/f). El sistema de lo devengado en la imputación de créditos y gastos del año fiscal. Derecho fiscal (tomo XIV). p. 181.

Jarach, D. (1998). Derecho tributario. Liceo Profesional CIMA: Earlies Eds.

Lagares, M. (s/f). La unidad contribuyente en el impuesto sobre la renta de las personas físicas. Hacienda Pública Española (n. 3). p. 69.

Millan, C. (1999). El mínimo exento en el sistema tributario español. Madrid: Marcial Pons. p. 71.

Peirano, J. (1962). Problemas del impuesto a la renta relacionados con el año fiscal. Semana Tributaria. Montevideo. p. 88.

Sanabria, R. (1997). Comentario al código tributario y los ilícitos tributarios. (3. a ed.). Perú: Edit. San Marcos.

Sanabria, R. (1991). Revista de Derecho de la Facultad de Derecho de la USMP. Lima. p. 157.

Superintendencia Nacional de Administración Tributaria (Sunat) (S/F). Disponible en: www.sunat.gob.pe.

Valdés, R. (1992). Instituciones del derecho tributario. Buenos Aires: Ediciones de Palma. 


\section{ANEXOS}

Tabla 1: Resultados según normatividad vigente

\begin{tabular}{|c|c|c|c|c|}
\hline Renta de 4ta. Categoría & Escala & Tasa & $\mathrm{N}^{\circ}$ Profesionales & $\%$ \\
\hline $\begin{array}{l}\text { Grupo 1:No alcanzan el monto } \\
\text { mínimo de sus rentas }\end{array}$ & No afecto & - & 28 & 56 \\
\hline \multirow{2}{*}{$\begin{array}{l}\text { Grupo 2: Alcanzan la primera } \\
\text { escala. }\end{array}$} & Primera escala & \multirow{2}{*}{$15 \%$} & \multirow{2}{*}{19} & \multirow{2}{*}{38} \\
\hline & 0 a 93,150 & & & \\
\hline \multirow{2}{*}{$\begin{array}{l}\text { Grupo 3:Alcanzan la Segunda } \\
\text { escala }\end{array}$} & Segunda escala & \multirow{2}{*}{$21 \%$} & \multirow{2}{*}{2} & \multirow{2}{*}{4} \\
\hline & 93,151 a 186,300 & & & \\
\hline \multirow{2}{*}{$\begin{array}{l}\text { Grupo } 4 \text { : Alcanzan la tercera } \\
\text { escala }\end{array}$} & Tercera escala & \multirow{2}{*}{$30 \%$} & \multirow{2}{*}{1} & \multirow{2}{*}{2} \\
\hline & 186,301 a más & & & \\
\hline & $N=$ & & 50 & 100 \\
\hline
\end{tabular}

Fuente: elaboración propia

Gráfico 1: Recaudación según normas vigentes

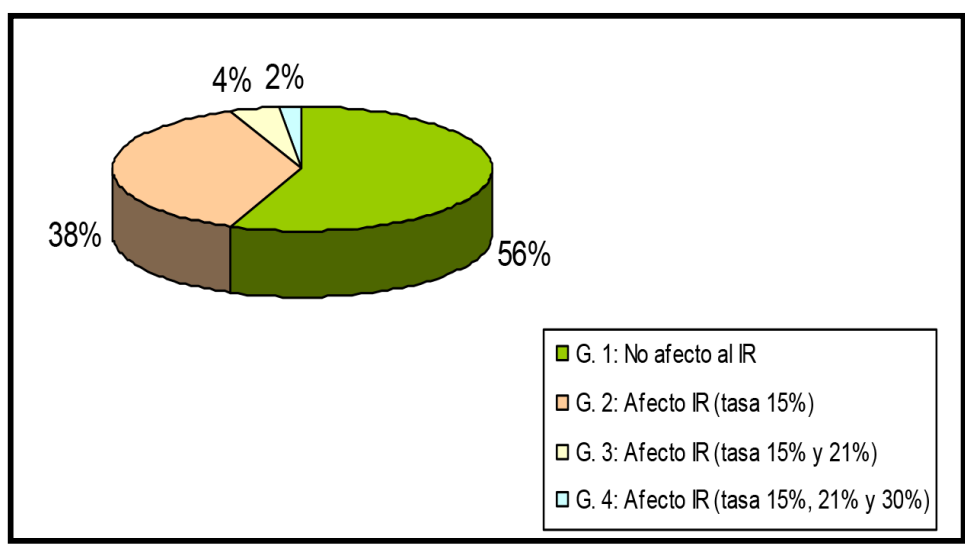

Fuente: elaboración propia 


\section{Cuadro 1}

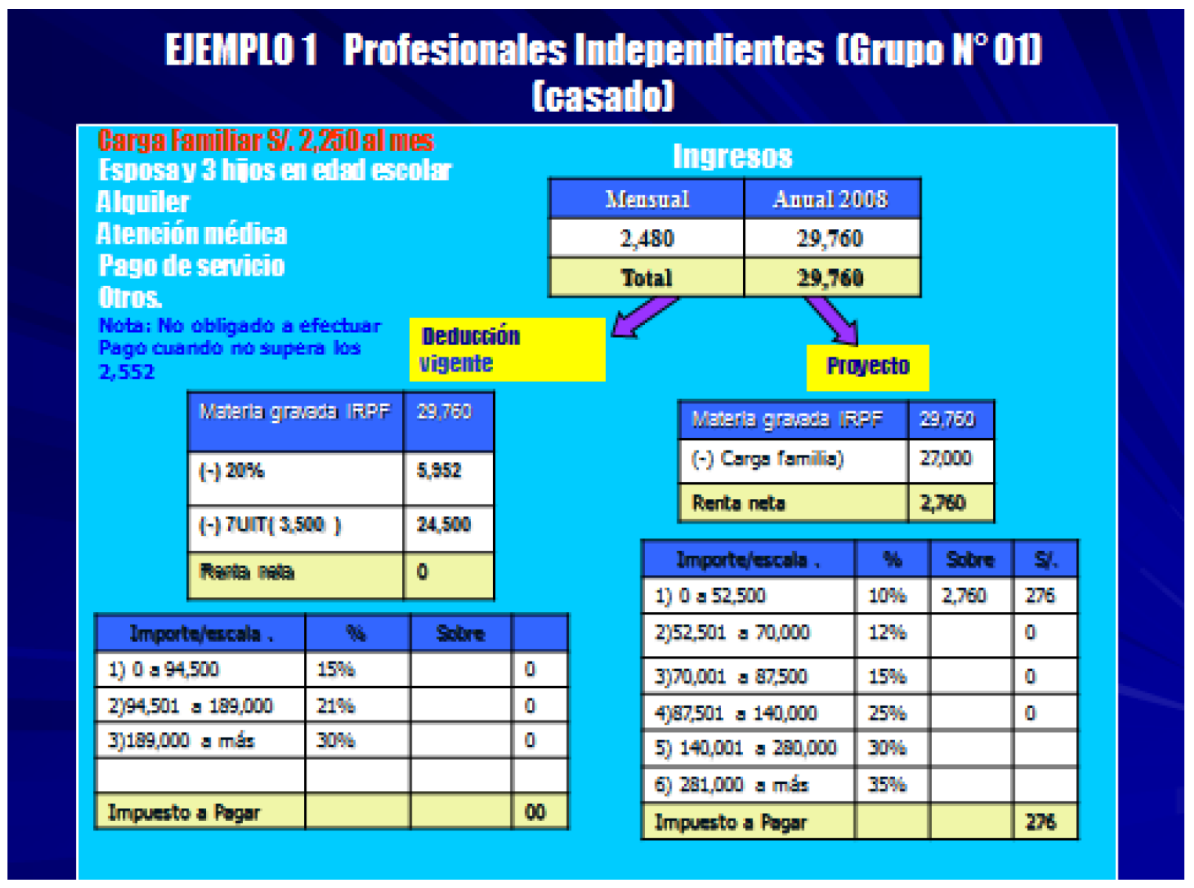

Fuente: elaboración propia

\section{Cuadro 2}

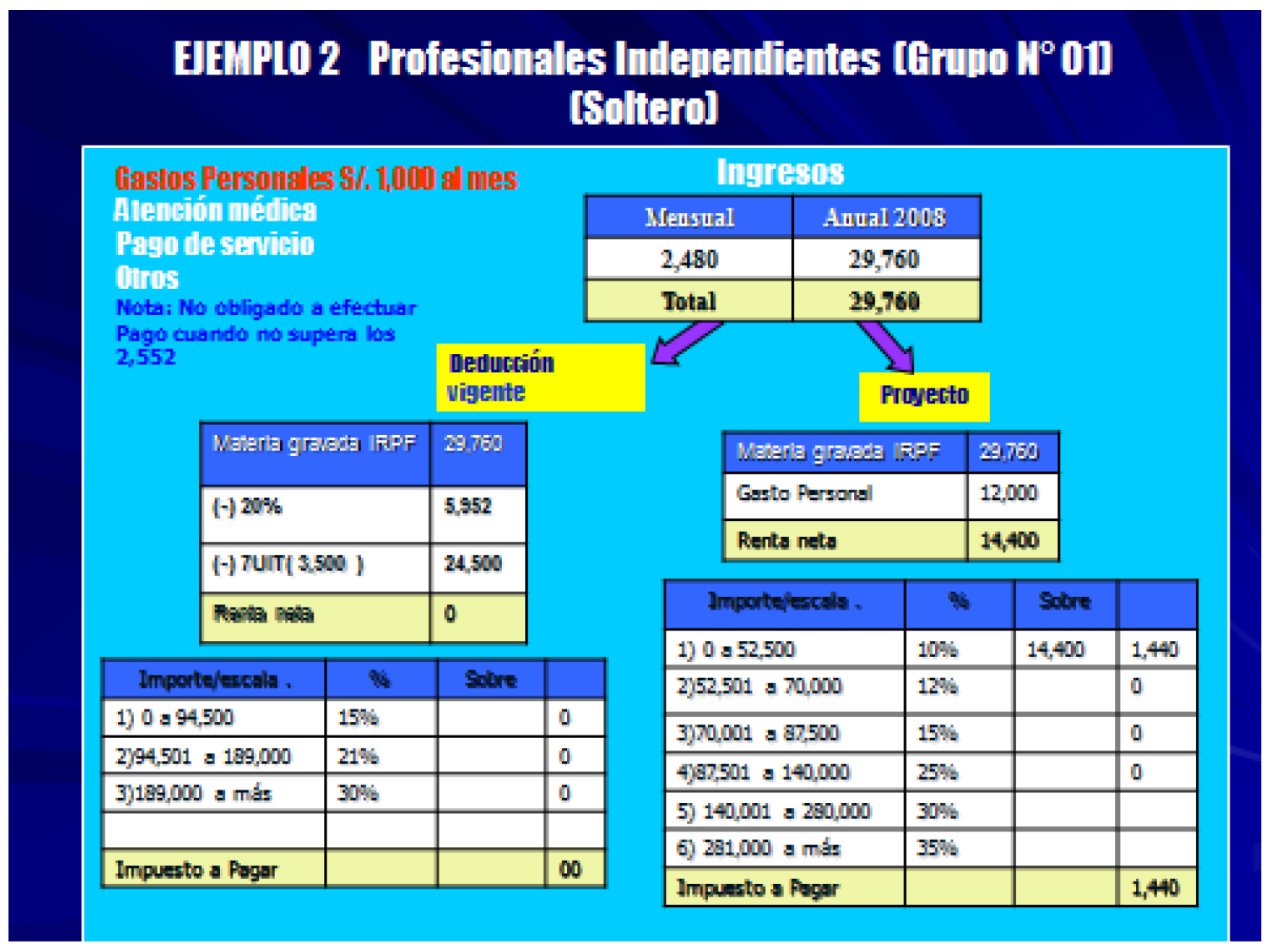

Fuente: elaboración propia 


\section{Cuadro 3}

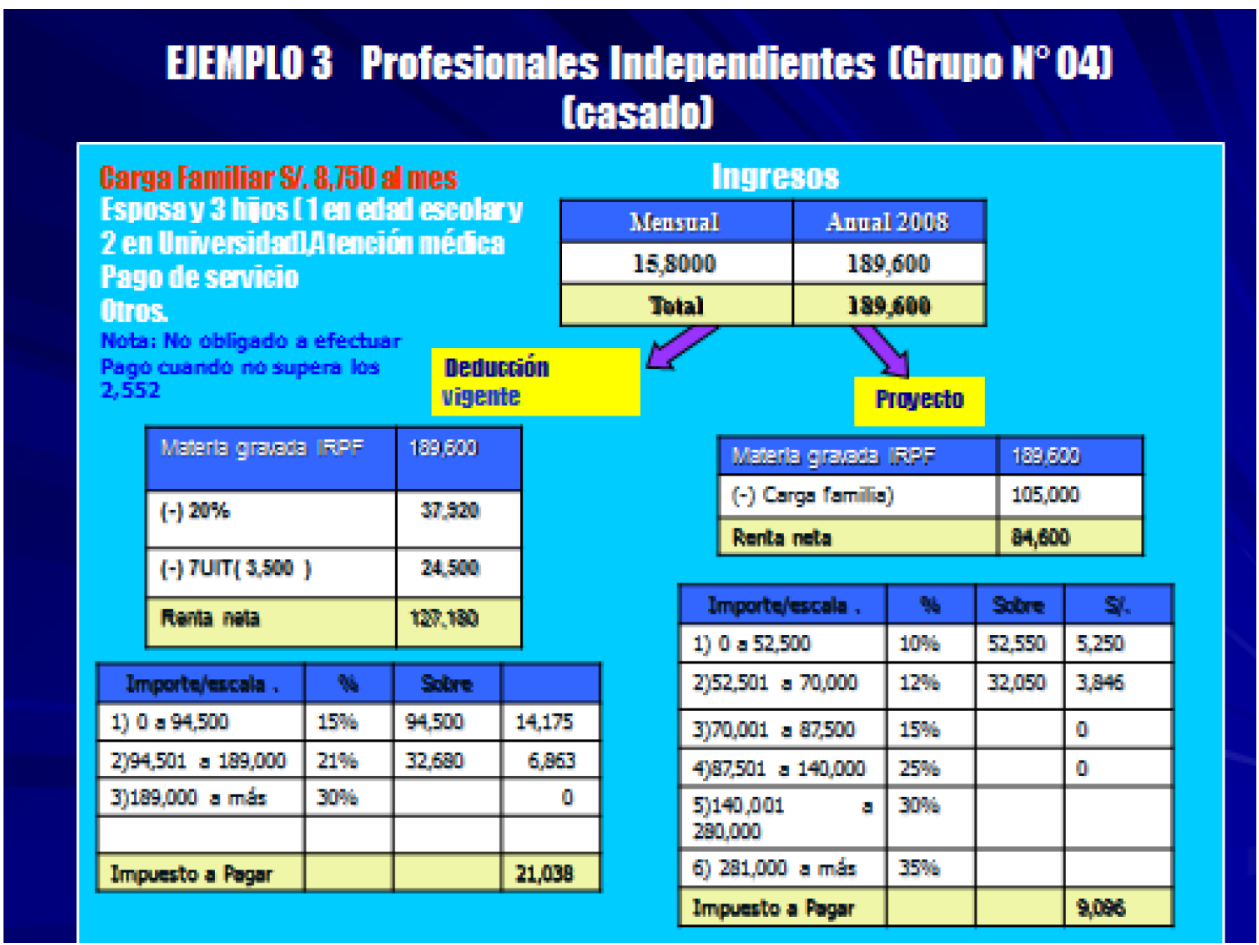

Fuente: elaboración propia

\section{Cuadro 4}

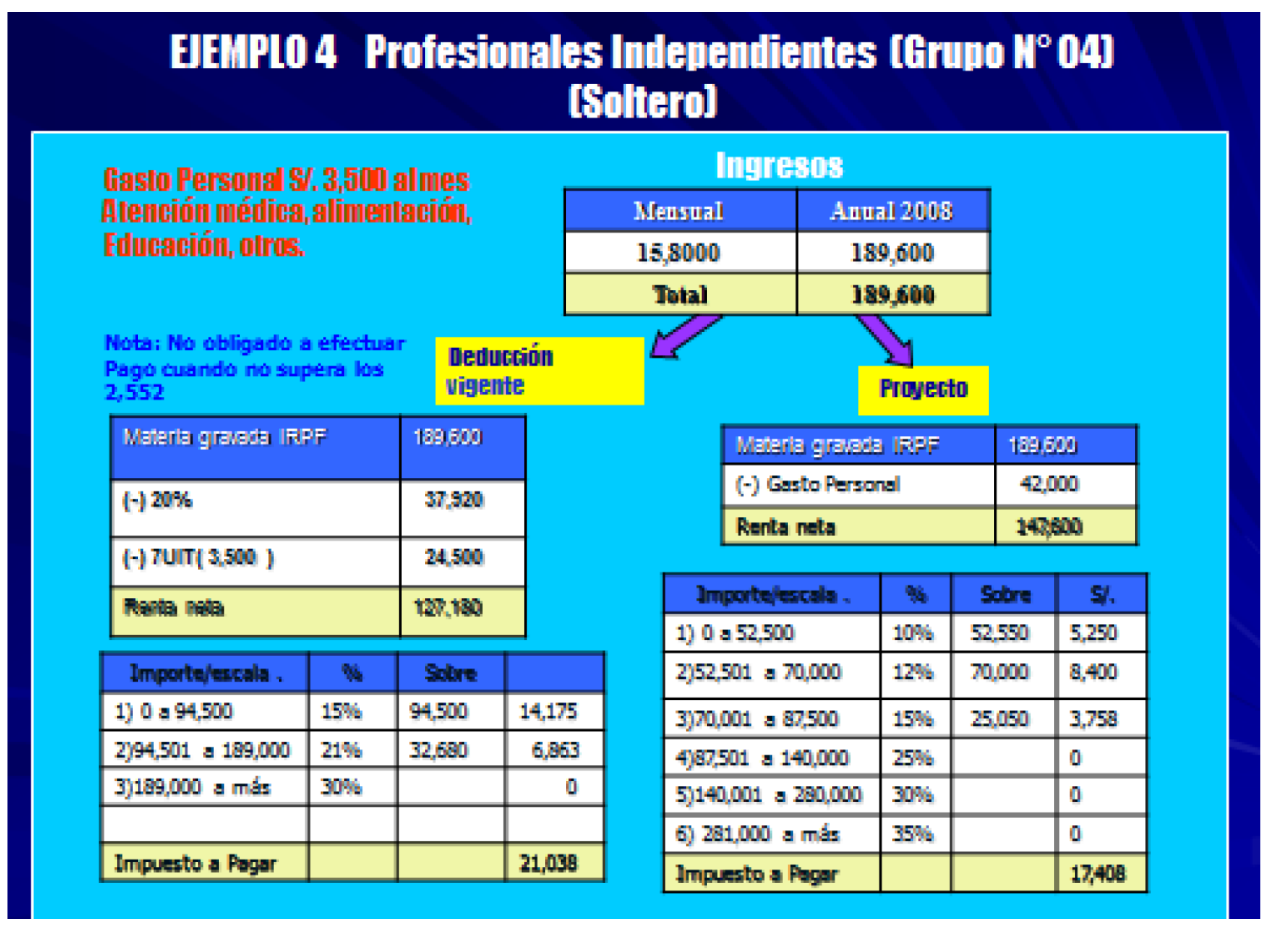

Fuente: elaboración propia 